\title{
Numerical simulation of resonant scattering of energetic electrons in the outer radiation belt
}

\author{
Yuto Katoh*, Takayuki Ono, and Masahide Iizima \\ Graduate School of Science, Tohoku University, Sendai, Japan
}

(Received July 15, 2003; Revised September 26, 2004; Accepted January 24, 2005)

\begin{abstract}
Resonant diffusion processes of high energy electrons are studied numerically by using an original simulation scheme where cold electrons are treated as a fluid and hot electrons are treated as particles including fully relativistic effects. The present simulation scheme enables us to investigate the resonant scattering process in the inner magnetosphere, which has been difficult to study by using the PIC code. We investigated the variation of the distribution of electrons through the resonant diffusion process by comparing the present numerical experiment and the quasi-linear theory under the same condition of the interaction between whistler-mode waves and high energy electrons. The difference between their results suggests the importance of the verification of the theory under the realistic plasma condition corresponding to the observed parameters in the outer radiation belt.
\end{abstract}

Key words: Radiation belt, numerical experiment, quasi-linear diffusion, relativistic electrons.

\section{Introduction}

The dynamic behavior of the radiation belt associated with geomagnetic storms has become one of the most interesting research subjects of the inner magnetosphere region after its discovery (Vampola and Korth, 1992; Blake et al., 1992; Baker et al., 1994). In the outer radiation belt, the flux of relativistic electrons decreases in the main phase of the geomagnetic storms and often increases again in the recovery phase (Selesnick and Blake, 2000; Obara et al., 2000; Meredith et al., 2002). The scenario of the flux increase of high energy electrons has been investigated theoretically and observationally on the basis of two diffusion processes; the one is energization due to the radial diffusion process, and the other is energy diffusion process due to wave-particle interaction with enhanced whistler chorus waves during the storm time.

An estimation based on the radial diffusion model implemented with a time dependent radial diffusion coefficient parameterized by $K_{p}$ was examined by Brautigam and Albert (2000). They have compared phase space density profiles with the result of the observation made by the CRRES and LANL satellites, and have concluded that the radial diffusion model does not by itself reproduce the increase of $\mathrm{MeV}$ electrons in the recovery phase. They have also suggested that the electron acceleration via wave-particle interaction possibly explains the inconsistency between the estimation of the theoretical model and the observation results. Similar discussion was made by Meredith et al. (2002). They compared the energy spectrum of energetic electrons

\footnotetext{
*Now at: Research Institute for Sustainable Humanosphere, Kyoto University, 611-0011, Japan.

Copyright (c) The Society of Geomagnetism and Earth, Planetary and Space Sciences (SGEPSS); The Seismological Society of Japan; The Volcanological Society of Japan; The Geodetic Society of Japan; The Japanese Society for Planetary Sciences; TERRAPUB.
}

at $\mathrm{L}=4.5$ observed by the CRRES spacecraft and the theoretical estimation given by Brautigam and Albert (2000) during the geomagnetic storm of October 9, 1990. They have shown that the fluxes estimated from the radial diffusion model are consistent with the observation at low energy part $E \lesssim 0.8 \mathrm{MeV}$; however, at higher energy part $E \gtrsim 0.8 \mathrm{MeV}$, the radial diffusion model can not explain the observed fluxes.

Furthermore, on the basis of the observations of the NOAA and EXOS-D (Akebono) satellites during the geomagnetic storm of November 3, 1993; Miyoshi et al. (2003) have pointed out that the radial profile of the phase space density forms a peak at $\mathrm{L}=4.0$ during the recovery phase, and that the spectral hardening of the electron flux occurs first near the inner edge of the outer belt. Such a radial profile of the phase space density has also been reported by the observations of the POLAR satellite (Selesnick and Blake, 2000). These results strongly supported the local acceleration hypothesis of the outer radiation belt electrons. Most of these workers think that the most promising candidate is the wave particle interaction process with the enhanced whistler-mode waves simultaneously observed during the recovery phase of geomagnetic storms.

In recent years, the acceleration process of the relativistic electrons has been studied in detail for the case of the stochastic acceleration caused by the resonant interaction with the storm-time enhanced whistler-mode waves (Summers and Ma, 2000; Miyoshi et al., 2003). Summers and Ma (2000) examined the rebuilding process of the energetic electrons in the outer radiation belt by using FokkerPlanck formulation, and Miyoshi et al. (2003) also proposed a model of the flux enhancement of $\mathrm{MeV}$ order electrons on the basis of the results of the observation of the Akebono and NOAA satellites. Their results were basically consistent with the in-situ observations which support the importance of the resonant scattering by the whistler- 


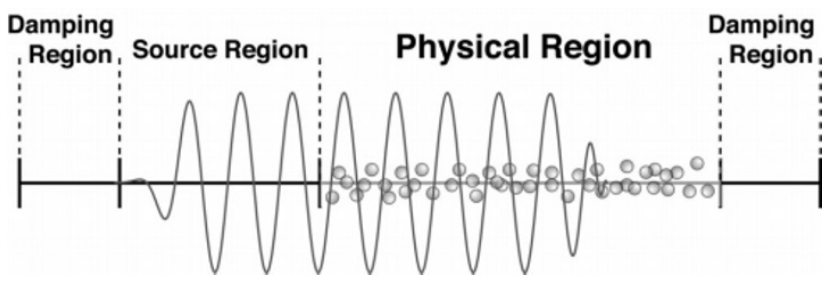

Fig. 1. Configuration of the simulation system. High energy electrons, which are treated as particles, are distributed only in the physical region. Plasma waves which are generated in the source region and propagate into the physical region, and are finally absorbed in the damping regions.

mode waves in the energy diffusion process (Meredith et al., 2002). However, a quantitative evaluation of the diffusion coefficients has not been fully understood and still remained as an important subject for the future studies because the time scale of the flux enhancement is essentially determined by the diffusion coefficients.

To understand the physical process of the energization of the radiation belt electrons, a numerical simulation is an useful tool to evaluate the effect of background plasma parameters and the non-linear effect of the wave-particle interaction. Such numerical approaches have been done by several workers by using test-particle analyses (e.g., Roth et al., 1999; Albert, 2000). Their trials have successfully shown that the effect of phase trapping is important in the electron energizing processes through the resonant interaction, although the energy transfers between waves and particles have not been included in their simulation model. The full-particle simulations are necessary for studying the interaction between particles and electromagnetic waves fully considering the effect of the energy exchange; however, the computational time and memory consumption prevent the execution of such numerical experiments. When we employ the PIC code, we should choose the grid size $\Delta x$ to be not much larger than the Debye length $\lambda_{e}$, as

$$
\Delta x \leq 3 \lambda_{e},
$$

to avoid a nonphysical instability caused by grid spacing (Birdsall and Langdon, 1985; Omura and Matsumoto, 1993). Therefore, the full particle treatment requires a large number of spatial grids to simulate the resonant interaction processes in the inner magnetosphere.

In the present paper, we examine the diffusion process of the relativistic electrons through the wave-particle interaction with the whistler-mode waves by using a newly developed simulation scheme, and compare the result with the diffusion rate derived from the quasi-linear approach. Our new simulation model, which can be categorized as a "electron hybrid code", allows us to investigate the resonant interaction processes of the relativistic electrons with electromagnetic plasma waves under various plasma environment including the energy transfer in the non-linear regime of the wave particle interaction. The differences between the numerical simulation and the quasi-linear theory and their implications are discussed.

\section{Electron Hybrid Model for Numerical Experi- ments}

When we study the resonant interaction between relativistic electrons and electromagnetic plasma waves in the outer radiation belt, we should treat a wide range of scale length; i.e., the wave length of the plasma waves is of the order of $10^{3}$ to $10^{4} \mathrm{~m}$, while the Larmor radius and the Debye length of cold electron, the medium of propagation of the plasma waves, is of the order of $10^{0} \mathrm{~m}$. Thus, in order to study the interaction by using a full particle simulation code, it is required that both the propagation of the plasma waves and the motion of the cold electrons should be considered at the same time and it is not realistic way of treatment as has been mentioned in the previous section.

Our newly developed simulation scheme is based on a model where the high energy electrons are treated as relativistic particles and the cold electrons are treated as a fluid, focusing on the differences in the spatial scales of their Larmor radii. Hereinafter, we refer to this hybrid model as "Electron Hybrid Model". Numerical studies by employing a similar type model have been proposed by Rathmann et al. (1978) and Omura and Matsumoto (1982), and have been named "Long-Time-Scale Code", and by Taguchi et al. (2001), given a name of "Hybrid Darwin Code". Compared with these established simulation model, Electron Hybrid Model has an advantage on the treatment of the plasma waves; Maxwell's equations are directly solved to compute the evolution of the electromagnetic field in time and space without any assumptions. Therefore, the Electron Hybrid Model is one of the most realistic models to investigate the energy exchange process between the high energy electrons and the plasma waves which are propagating in a medium governed by the cold electron plasma.

Basic equations used in the present simulation scheme are as follows:

$$
\begin{aligned}
\frac{\partial \boldsymbol{v}_{f}}{\partial t} & =-\left(\boldsymbol{v}_{\boldsymbol{f}} \cdot \boldsymbol{\nabla}\right) \boldsymbol{v}_{\boldsymbol{f}}+\frac{q}{m}\left(\boldsymbol{E}+\boldsymbol{v}_{\boldsymbol{f}} \times \boldsymbol{B}\right), \\
\frac{\partial \rho_{f}}{\partial t} & =-\boldsymbol{\nabla} \cdot\left(\rho_{f} \boldsymbol{v}_{f}\right), \\
\frac{\partial \boldsymbol{B}}{\partial t} & =-\boldsymbol{\nabla} \times \boldsymbol{E} \\
\frac{\partial \boldsymbol{E}}{\partial t} & =\frac{1}{\mu_{0} \varepsilon_{0}} \boldsymbol{\nabla} \times \boldsymbol{B}-\frac{1}{\varepsilon_{0}} \boldsymbol{J}, \\
\frac{d \boldsymbol{p}_{\boldsymbol{p}}}{d t} & =q\left(\boldsymbol{E}+\boldsymbol{v}_{\boldsymbol{f}} \times \boldsymbol{B}\right), \\
\frac{d \mathcal{E}_{k i n}}{d t} & =\boldsymbol{v}_{\boldsymbol{f}} \cdot \frac{d \boldsymbol{p}_{\boldsymbol{p}}}{d t}
\end{aligned}
$$

and

$$
\boldsymbol{p}_{\boldsymbol{p}}=\frac{\boldsymbol{p}_{\boldsymbol{p}}}{c^{2}} \mathcal{E}_{k i n}
$$

where subscripts $f$ and $p$ denote the quantities associated with the cold (fluid) electron and the hot (particle) electron, respectively. $\mathcal{E}_{k i n}$ is the kinetic energy of hot electron including its rest mass energy. We compute the current from the motion of both cold and high energy electrons. To implement the simulation code, each physical values are normalized to be dimensionless quantities; time is normalized 
(a) Initial Velocity Distribution

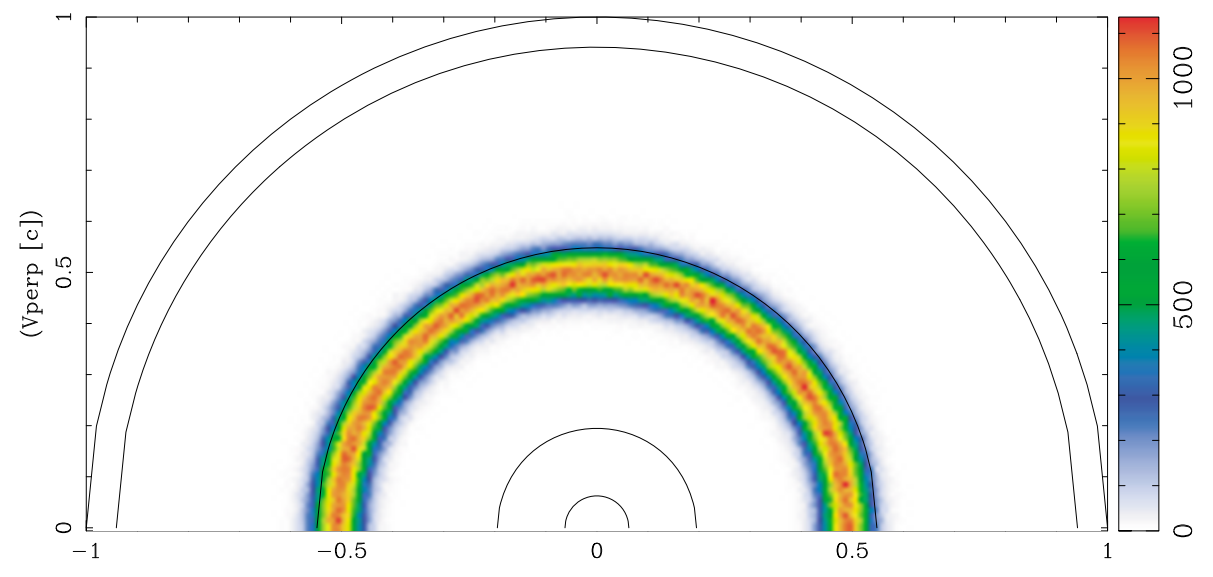

(b)

(Vpara $[\mathrm{c}])$

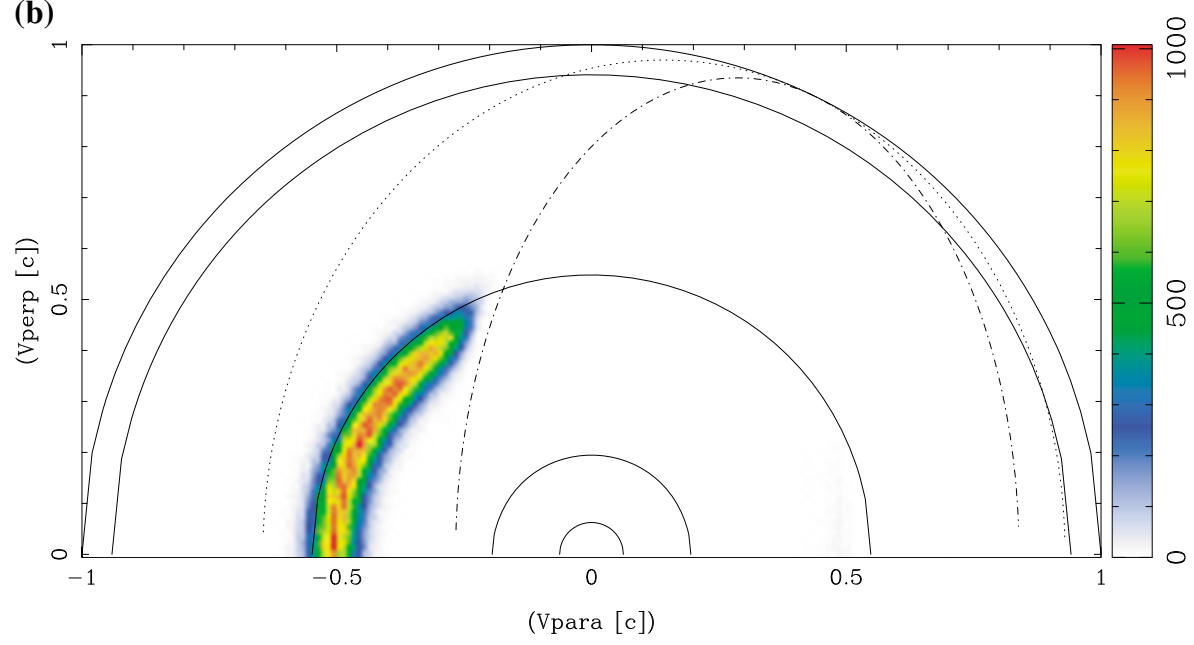

Fig. 2. (a) Initial velocity distribution of energetic particles in the phase space. Five thin lines in each figure denote constant energy lines of 1,10 , 100 $\mathrm{keV}, 1 \mathrm{MeV}$ and $\infty(v=c)$, respectively. (b) Distribution of resonant particles in phase space. As for a reference, resonance curves are also shown for whistler-mode waves with the frequency of 0.3 (dash-dotted line) and 0.6 (dashed line) $\Omega_{e}$ for the case $f_{p} / f_{c}=1.0$.

by the electron cyclotron frequency $f_{c e}$, velocity and length are normalized by the speed of light $c$ and $c / f_{c e}$, respectively.

Simulation system is separated into three regions as shown in Fig. 1; namely, the physical region, the wave source region, and the damping region. We assume that the simulation system is one dimension and aligned with the background magnetic field direction. The three components of electromagnetic field and motions of electron are computed numerically. The high energy electrons are distributed in the physical region by employing a periodic boundary condition. To avoid the reflection of the waves near the boundary of the physical region, the masking method is applied to the electric field caused by the current density of the hot electrons; because the location of the boundary of the hot electrons is different from that of the cold electrons. By setting the wave source region at the external space of the physical region, we treated the whistlermode waves propagating into the physical region from the external source along the direction of $\mathrm{X}$-axis.

In the present simulation, we choose the system length of the physical region $\mathrm{L}_{x}$ as $4096 \Delta x$ aligned to the initial magnetic field direction while grid spacing $\Delta x$ is set to be as $1.0 \times 10^{-2} c / f_{c e}$ and time step $\Delta t$ as $7.5 \times 10^{-3} f_{c e}^{-1}$, which satisfy the time and spatial limits to represent the propagation of the whistler-mode waves. The plasma density and the magnetic field strength are set to be as $f_{p} / f_{c}=1.0$; considering the parameter at the outside region of the plasmapause. The grid spacing and the time step, then, corresponding to $100 \mathrm{~m}$ and $0.268 \mu \mathrm{sec}$, respectively. In the next section, we carry out the numerical experiment to investigate the interaction process between the high energy electrons and the whistler-mode waves. We assume that the high energy electrons are uniformly distributed in the physical region, having a distribution function of a shifted bimaxwellian type distribution centered at a half of the speed of light, corresponding to the energy of $79 \mathrm{keV}$, whereas their initial pitch angles and gyrophases are given to form an isotropic distribution (Fig. 2(a)). The number density of high energetic electrons is set to be $10^{-6}$ of the background cold electrons; 1,310,720 super-particles have been used for the calculation of the motion of high energy electrons. The frequency spectrum of the whistler-mode waves is given as gaussian function which is continuously distributed from 


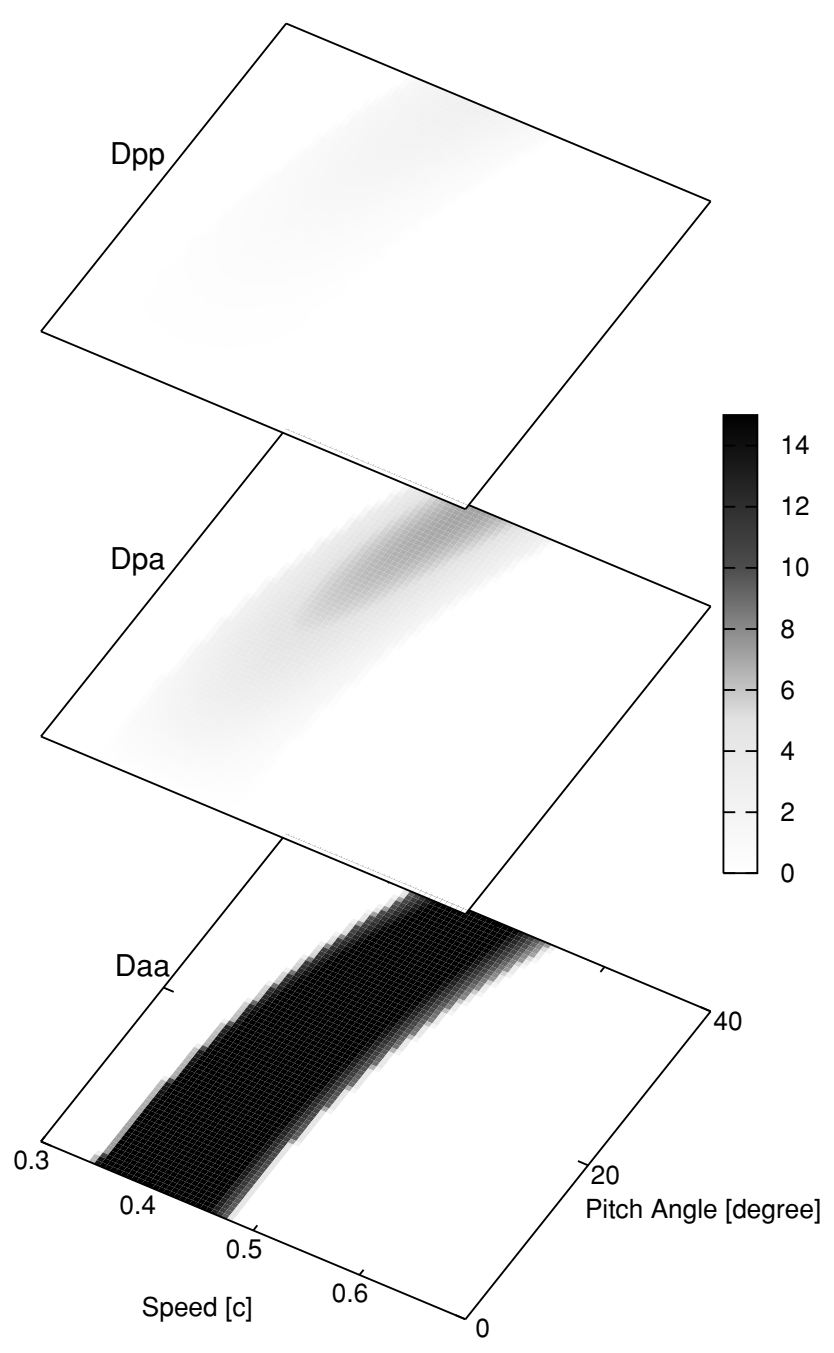

Fig. 3. Diffusion coefficients used in the numerical check. The color index represents the value of the normalized coefficients. From top to bottom, $D_{p p}, D_{p \alpha}\left(=D_{\alpha p}\right)$ and $D_{\alpha \alpha}$ are represented in the $|v|-\alpha$ plane using a same color index.

0.3 to $0.6 \Omega_{e}$; and their effective value of wave amplitude is set to be $1 \mathrm{mV}$ rms $/ \mathrm{m}$. Under these settings, we perform each experiments up to $7.5 \times 10^{3}$ gyro-cycle of electrons which corresponding to $270 \mathrm{msec}$ time interval in the real-space.

\section{Simulation Results}

The distribution of the interacting particles in the phase space at the end time of the experiment is shown in Fig. 2(b), where the interacting electrons are picked up with a criterion that they show the variations from the initial values of speeds and pitch angles more than $0.1 \%$ and 0.1 degree, respectively. The present simulation result shows that the interacting electrons are distributed very close to the cyclotron resonance curves, which are expressed by $\omega-k_{\|} \mathbf{v}_{\|}=\Omega_{e} / \gamma$, where $\mathbf{v}_{\|}$is the field aligned component of the velocity of the resonant particles and $\gamma$ is the Lorentz factor, respectively. This result suggests that the interaction process mainly occurs as resonant interaction and this process has been accurately simulated in our model. To see the diffusion rate in the resonant interaction process, the deviations from the initial velocity and the pitch-angle of each electrons have been investigated. As will be discussed later in detail, the velocity distribution of the high energy electrons is smoothed by resonant scattering process.

\section{Quasi-linear Approach of the Diffusion Process}

As for the next step, we examine the diffusion process of resonant particles by using the quasi-linear diffusion equation under the same parameter set as used in the numerical experiment. The quasi-linear diffusion equation is given by;

$$
\begin{aligned}
\frac{\partial f_{0}^{l}}{\partial t}= & \frac{1}{p \sin \alpha} \frac{\partial}{\partial \alpha} \sin \alpha\left(D_{\alpha \alpha} \frac{1}{p} \frac{\partial f_{0}^{l}}{\partial \alpha}+D_{\alpha p} \frac{\partial f_{0}^{l}}{\partial p}\right) \\
& +\frac{1}{p^{2}} \frac{\partial}{\partial p} p^{2}\left(D_{p \alpha} \frac{1}{p} \frac{\partial f_{0}^{l}}{\partial \alpha}+D_{p p} \frac{\partial f_{0}^{l}}{\partial p}\right),
\end{aligned}
$$

where $f_{0}^{l}$ is spatially uniform, zeroth-order distribution function for a plasma species $l, \alpha$ refers pitch angle, and $p$ denotes momentum including relativistic effect, respectively. The nonrelativistic form of the diffusion equation has been given by Lyons (1974) in the spherical coordinates in $(v, \alpha)$ space. Its bounce-averaged equation is well-known and has been solved by several workers (e.g. Lyons et al., 1973; Kozyra et al., 1995); however, to average the bouncing motion is not proper for comparing with the simulation result. Therefore we directly solve Eq. (9) numerically by using simple centered-difference method.

The diffusion coefficients in the calculation of Eq. (9) have been estimated by using the same procedure as has been given by Albert (1999), which was based on the formula derived by Lyons (1974). In this method, the diffusion coefficients for the electron of the velocity $v$ and the pitch angle $\alpha$ are calculated by assuming the wave spectrum to be a gaussian form with the frequency range from $\omega_{L C}$ to $\omega_{U C}$ (peak at $\omega_{m}$ ). That is, the spectrum of the whistler-mode wave is defined by the function $\Gamma(\omega)$, given by

$$
\Gamma(\omega)=\frac{\exp \left(-\left(\omega-\omega_{m}\right)^{2} / \delta \omega^{2}\right)\left(\Omega_{0} / \delta \omega\right)}{\operatorname{erf}\left(\left(\omega_{\mathrm{UC}}-\omega_{m}\right) / \delta \omega\right)+\operatorname{erf}\left(\left(\omega_{m}-\omega_{\mathrm{LC}}\right) / \delta \omega\right)},
$$

where $\Gamma(\omega)$ is the function of $\omega_{U C}, \omega_{L C}$ and $\omega_{m}$. When these wave spectrum parameters as well as the wave normal angle $\theta$ and the order of the resonance condition $\mathrm{n}$ are given, the diffusion coefficients can be evaluated in the $v-\alpha$ space. The calculation of Eq. (9) is then carried out using these coefficients.

\subsection{Evaluation of the quasi-linear diffusion process}

Since the numerical scheme for the calculation of Eq. (9) is also originally developed in this work, a verification has been performed concerning a basic process of quasi-linear diffusion, i.e., the relaxation of a bump on the velocity distribution during the resonant interaction. In the calculation for verification, we assume that the pitch angle distribution is isotropic whereas the velocity distribution $f(v)$ has a form as

$$
f(v)=C_{1} \exp \left\{-\frac{(v)^{2}}{v_{a 1}^{2}}\right\}+C_{2} \exp \left\{-\frac{\left(v-v_{d}\right)^{2}}{v_{a 2}^{2}}\right\}
$$

where $C_{1}$ and $C_{2}$ are constants. We have set $v_{d}=0.48 c$, $v_{a 1}=0.27 c$ and $v_{a 2}=0.05 c$. The spectrum of the whistlermode wave $\Gamma(\omega)$ has been set by Eq. (10) where $\omega_{U C}=$ 
(a)

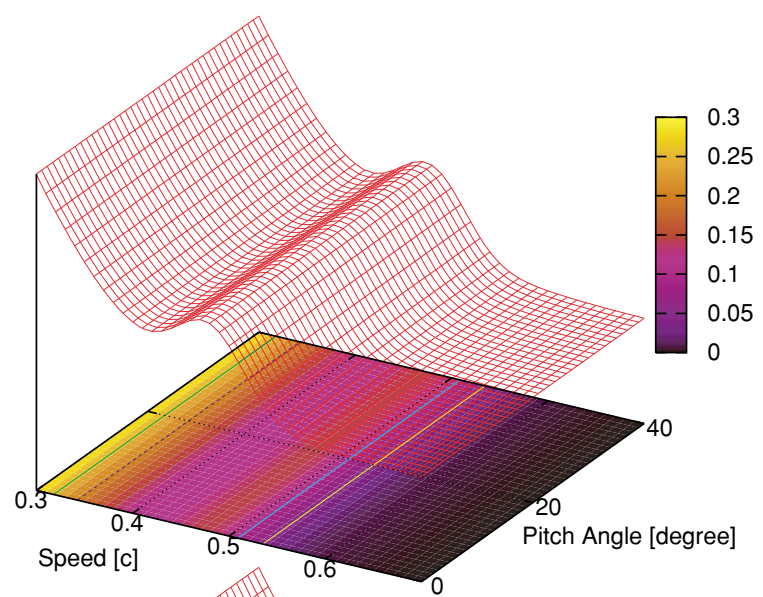

(b)

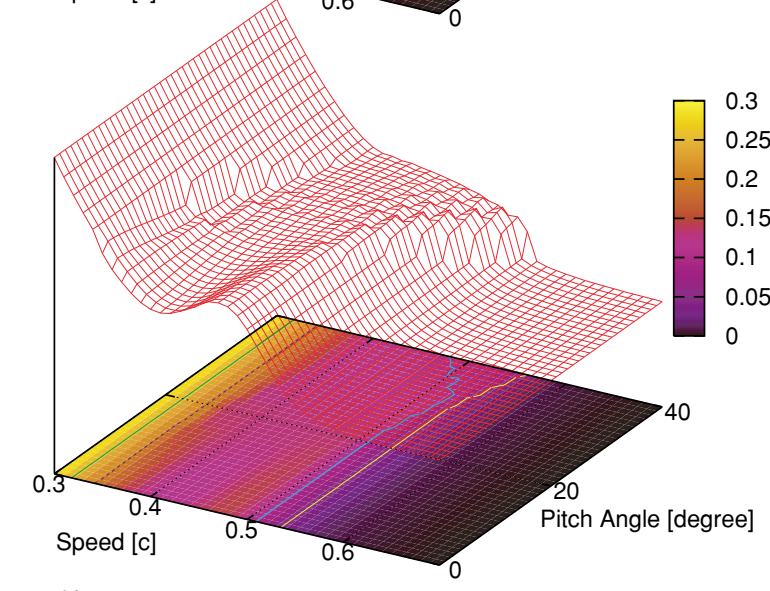

(c)

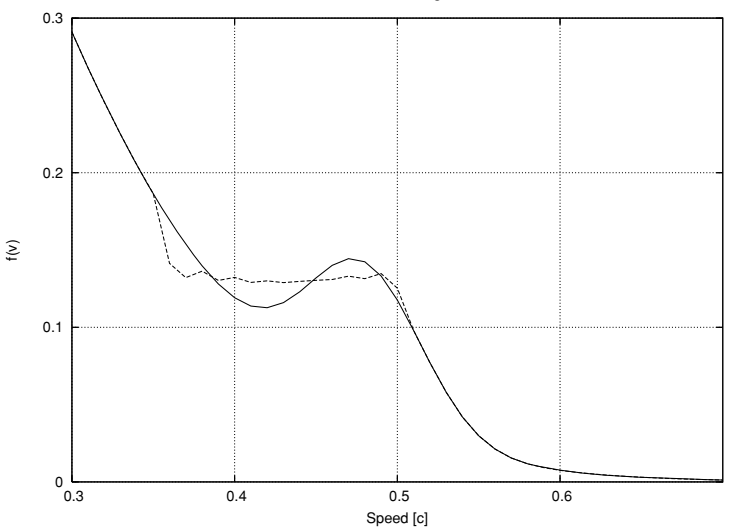

Fig. 4. Distribution function of electron in the $v-\alpha$ space at (a) $t=0$ and (b) $t=3.75 \times 10^{6} T_{c e}$, and (c) cross-section of velocity distribution with the pitch angle $\alpha=20^{\circ}$ at $t=0$ (solid line) and $t=3.75 \times 10^{6} T_{c e}$ (dashed line).

$0.55 \Omega_{e}, \omega_{L C}=0.45 \Omega_{e}$ and $\omega_{m}=0.5 \Omega_{e}$. Here we assume $n=-1$ and $\theta \sim 0^{\circ}$. These parameters have been chosen for a efficient resonant interaction with the electrons in the bump in the distribution with relatively large diffusion coefficients as shown in Fig. 3. Figure 3 shows the diffusion coefficients normalized by $B_{\text {wave }}^{2} / B^{2}\left(m_{e} v\right)^{2} \Omega_{e}$, where $B_{\text {wave }}$ represents the amplitude of wave magnetic field. Within the four components of the diffusion coefficient, the mixed term $D_{\alpha p}$ and $D_{p \alpha}$ often have negative value. In general, it is well known that a negative coefficient in the diffusion equation causes the differential equation to be unstable and the calculation of Eq. (9) to be difficult. In the present case, however, the mixed coefficients are almost positive and our

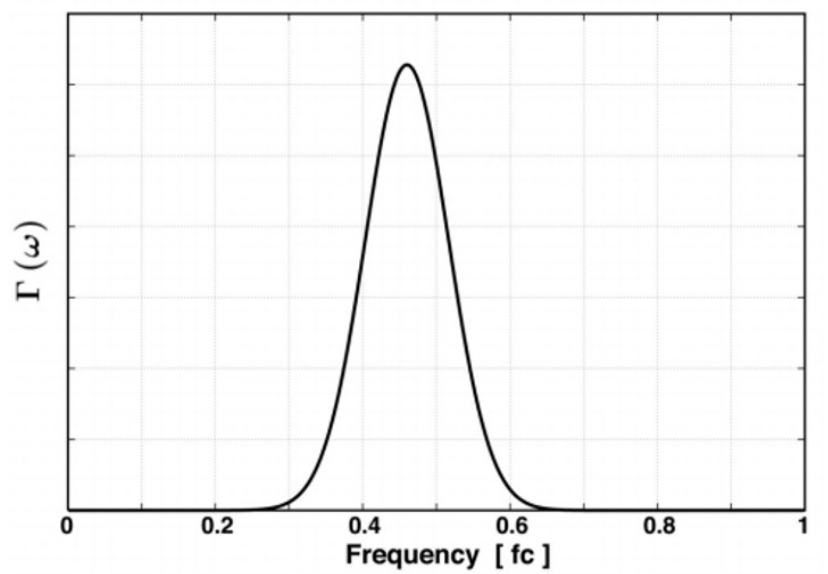

Fig. 5. Frequency spectrum $\Gamma(\omega)$ of the whistler-mode waves corresponding to the wave parameters used in the simulation and the estimation of diffusion rates by quasi-linear diffusion equation. Frequency of the waves are normalized by nonrelativistic cyclotron frequency of electron.

calculation has been stably carried out. We have estimated, then, each coefficient within a velocity range from $0.3 \mathrm{c}$ to $0.7 \mathrm{c}$ and a pitch angle range from $0^{\circ}$ to $90^{\circ}$, focusing on the variation of the bump in the velocity distribution. To carry out the calculation of Eq. (9), the time step and the grid width of the velocity and the pitch-angle distribution are chosen to be $7.5 T_{c e}, 0.01 \mathrm{c}$ and $1^{\circ}$, respectively.

Figure 4(a) and (b) represent the distribution of electron at the initial condition and at $3.75 \times 10^{6} T_{c e}$, respectively. As shown in Fig. 4(b), the bump in the velocity distribution of electron is relaxed at $3.75 \times 10^{6} T_{c e}$ and shows a state of quasi-liner plateau. In the test calculation, the normalized diffusion coefficient $D_{p p} /\left(\left(B_{\text {wave }} / B\right)^{2}(m v)^{2} \Omega_{e}\right)=1.0$ at the pitch angle $\alpha=20^{\circ}$ and $v / c \sim 0.45$. Since we assumed that the intensities of background wave magnetic field and wave magnetic field are $1000 \mathrm{nT}$ and $10 \mathrm{pT}$ in the test calculation, $B_{\text {wave }} / B$ is equal to $10^{-5}$. The result of the test calculation showed that the distribution is relaxed at $T_{\text {final }}=3.75 \times 10^{6} T_{c e}$, therefore $\left(D_{p p} / p^{2}\right) \cdot T_{\text {final }}=$ $2.36 \times 10^{-3}$. Although this estimation is slightly small, we should take into account that the variation of the distribution is about 0.015 as shown in Fig. 4. Moreover, the temporal evolution of the distribution $\partial f / \partial t$ should be evaluated from both diffusion coefficients and inclination of the gradient of the distribution, which over unity in the initial condition of the present calculation. Based on these estimations, we found that the result of the test calculation of the quasilinear diffusion equation is acceptable. This result shows that our calculation has an enough validity to discuss the time evolution of Eq. (9).

\subsection{Comparison of diffusion processes expressed by the quasi-linear theory and the numerical simula- tion}

By using the scheme described in the previous section, we compared the diffusion processes determined by the quasi-linear diffusion equation and computed by the numerical simulation. In this case, we have selected the same wave parameters and the same distribution function of high energy electrons for both estimations of diffusion processes. 


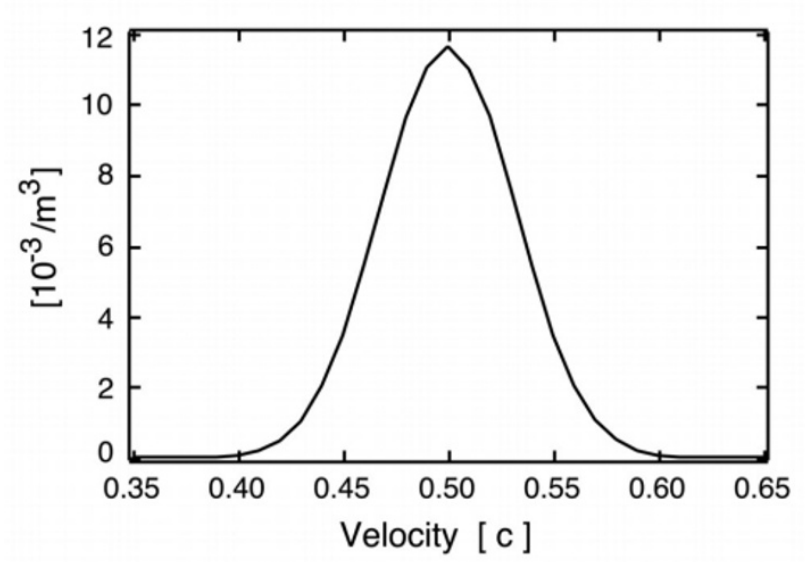

Fig. 6. Initial distribution function of the energetic electrons used in the numerical experiment. This function is also used in the estimation of the diffusion rates by quasi-linear diffusion equation.

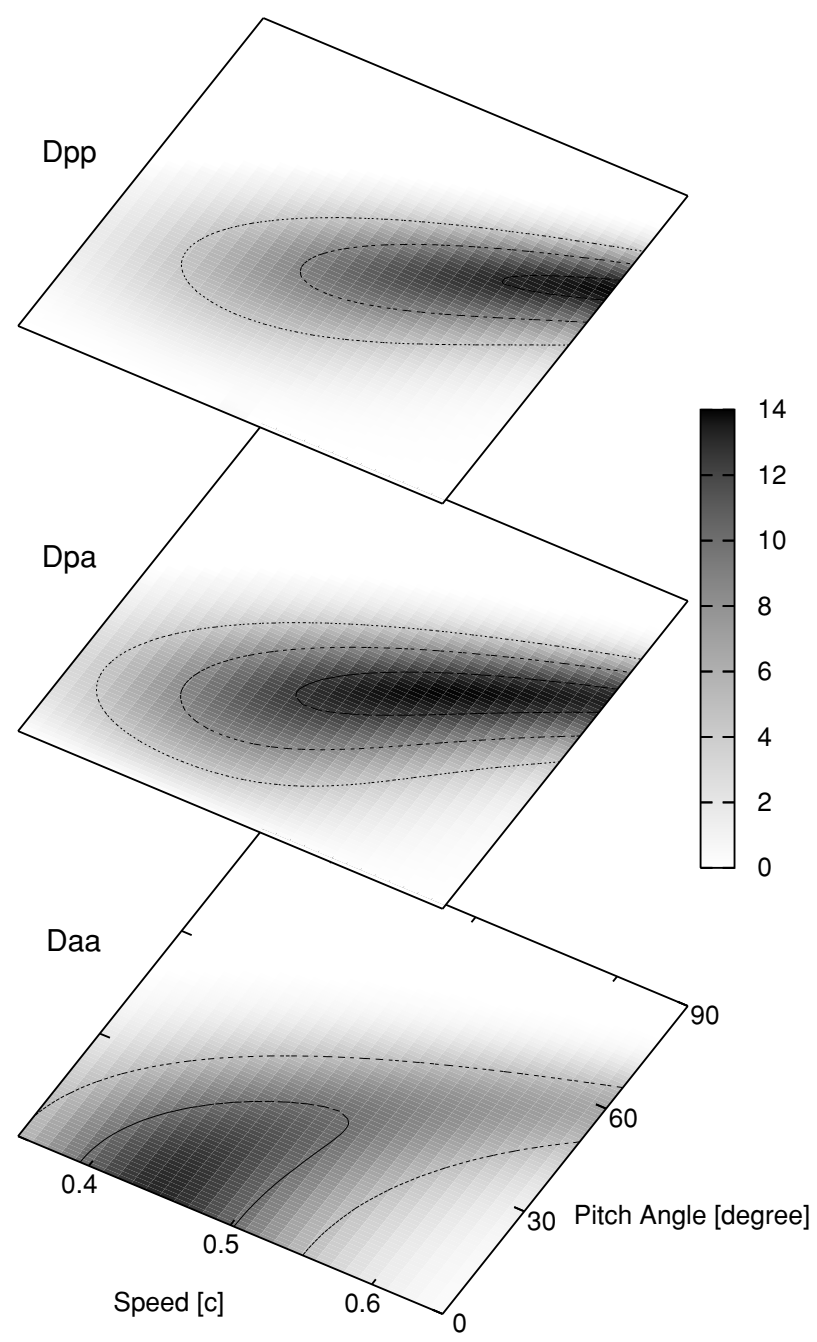

Fig. 7. Diffusion coefficients estimated for the same parameters used in the numerical simulation. From top to bottom, $D_{p p}, D_{p \alpha}\left(=D_{\alpha p}\right)$ and $D_{\alpha \alpha}$, respectively.

The wave spectrum and the particle distribution are shown in Figs. 5 and 6, respectively. Figure 5 indicates the shape of the function $\Gamma(\omega)$ given by Eq. (10) using the wave parameters: $\omega_{U C} / \Omega_{e}=0.62, \omega_{L C} / \Omega_{e}=0.3, \omega_{m} / \Omega_{e}=0.46$,

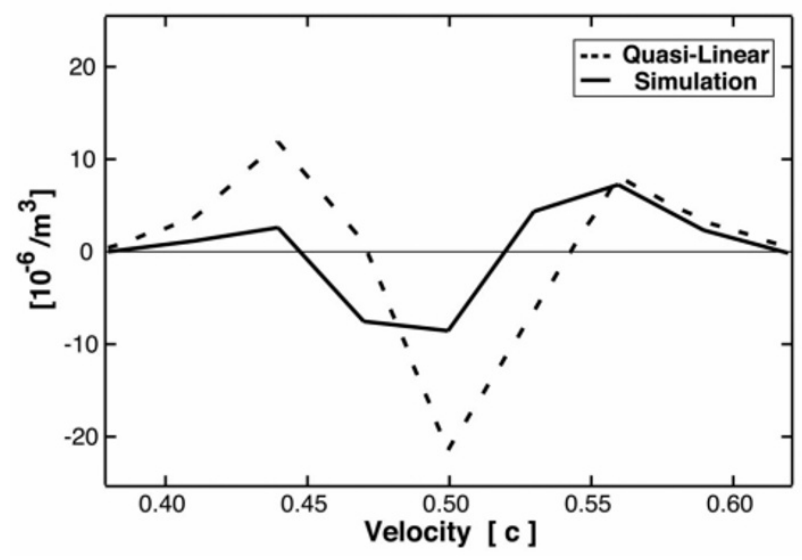

Fig. 8. Variations of the velocity distribution functions obtained by the present of simulation (solid line) and the quasi-linear diffusion equation (dashed line) during $7500 T_{c e}$.

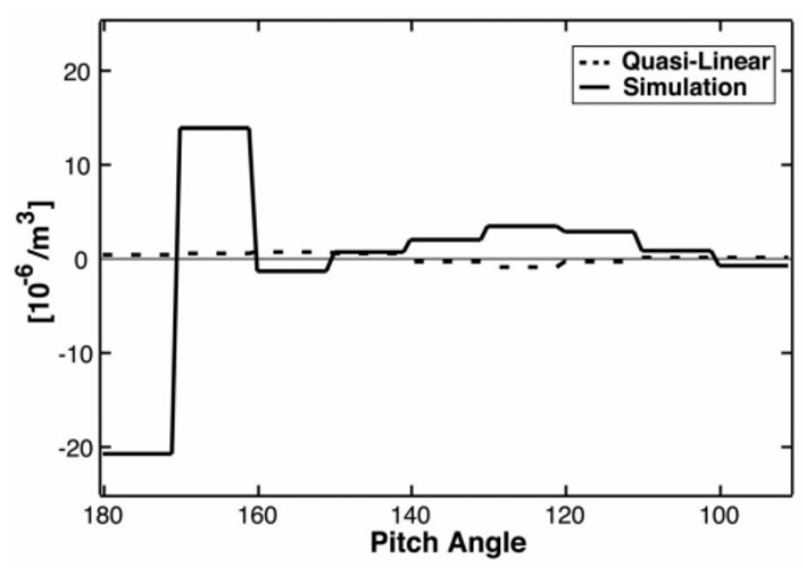

Fig. 9. Variations of the pitch angle distributions obtained by the simulation (solid line) and the diffusion equation (dashed line) during 7500 $T_{c e}$.

$\theta \sim 0^{\circ}$ and $n=-1$. Then, each diffusion coefficient has been calculated within a velocity range from $0.35 \mathrm{c}$ to $0.65 \mathrm{c}$ and a pitch-angle range from $0^{\circ}$ to $90^{\circ}$ (equivalent to $180^{\circ}$ to $90^{\circ}$ ), corresponding to the distribution of the high energy electrons as shown in Fig. 6. The estimated diffusion coefficients are plotted in Fig. 7.

A comparison of diffusion processes calculated by the quasi-linear diffusion equation and by the numerical simulation is given in Figs. 8 and 9. Dashed-lines and the solid lines on both Figs. 8 and 9 show the variation of the velocity and the pitch-angle distribution at the time of $7500 T_{c e}$ estimated by quasi-linear diffusion equation and the numerical simulation, respectively. These results reveal that the diffusion rates estimated by the quasi-linear diffusion equation are slightly different from those obtained by the numerical experiment. By comparing these results, it has been shown that the change of the distribution function around the center of Maxwellian distribution shows a similar tendency for both results; that is, the distribution function is smoothed due to the effect of the resonant diffusion, especially at high energy side where a magnitude of the variation of the distribution shows $7.2 \times 10^{-6}\left(\mathrm{~m}^{-3}\right)$ at $v \simeq 0.56$. However, 
there is a quantitative difference between them; that is, the variation of the distribution function obtained by the diffusion equation is five times as much as that of simulation at $v \simeq 0.44$, and twice at $v \simeq 0.5$. Moreover, a significant difference appears in the variation of the pitch angle distribution between results by the simulation and by the diffusion equation as shown in Fig. 9. In the simulation result, the electrons with a pitch angle range of $180^{\circ} \sim 170^{\circ}$ tend to diffuse into $170^{\circ} \sim 160^{\circ}$ region. This result suggests that a strong pitch angle scattering takes place for the electrons with the pitch angle range of $\sim 180^{\circ}$. On the other hand, no pitch angle scattering is occurs in the solution of the diffusion equation.

\section{Discussion and Summary}

In the previous section, we have shown that both the energy and the pitch angle diffusion obtained by the present simulation differ from those expected from the quasi-linear estimation, especially for the variation of the pith angles. To understand this difference, we think several effects described below should be discussed.

The first is an effect of nonlinearity which is not included in the quasi-linear theory, such as equivalent collisions between the whistler-mode waves and the high energy particles. To examine the effect of nonlinearity, a test simulation is performed where the whistler-waves have a banded spectrum which is continuously distributed from 0.5 to 0.8 $\Omega_{e}$. In this case, small pitch angle electrons do not exist near the resonant ellipse of the whistler-mode wave. If the pitch angle scattering of the high energy electrons takes place mainly by the intense nonlinear effect, it is expected that electrons are scattered being not related to the location of the resonant ellipse in the velocity space. Figure 10 shows the distribution of the resonant electrons in the phase space; the resonant electrons are selected using the same criterion as in Fig. 2(b). It is shown that the resonant scattering takes place merely on and close to the resonant ellipses of whistler-mode waves. Although the amount of the scattered electrons is small, almost all of the electrons which have negative $v_{\|}$are scattered due to the resonant interaction with self-excited whistler-mode waves (wave amplitude of several tens $\left.\mu \mathrm{V}_{\mathrm{rms}} / \mathrm{m}\right)$. Thus, even if the effect of the nonlinearity is evidently included in the present simulation, the electrons with small pitch angle are little influenced by the nonlinear effect under the present time scale.

Here we discuss unphysical effect comes from the boundary condition assumed in the numerical simulation. As shown in Fig. 1, we assumed that boundaries of particles and electromagnetic field is placed at different position. In this condition, particles crossing the boundary of the physical region will see a discontinuity in the fields; it should cause unphysical effect on the motion of particles, especially with small pitch angle particles. When we checked trajectories of small pitch angle particles, the significant unphysical effect on the motion of these particles was not appeared in the snap-shot of the simulation. However, the effect of the discontinuity may be significant by cumulating a number of boundary crossing even if the effect of the boundary is a little; there may be the unphysical effect due to the boundary condition concerning the difference at the

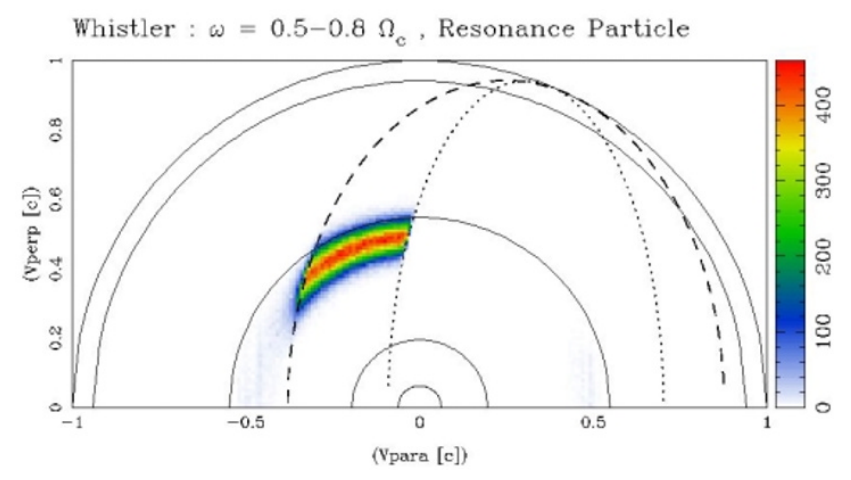

Fig. 10. Distribution of scattered particles in the phase space in a case where the wave has of a banded spectrum which is continuously distributed from 0.5 to $0.8 \Omega_{e}$. Resonant ellipses for the case of the wave frequencies, 0.5 (dashed line) and $0.8 \Omega_{e}$ (dotted line) with the plasma parameter of $f_{p} / f_{c}=1.0$ are also shown.

small pitch angle range in Fig. 9. On the other hand, when we consider that the discontinuity of the wave field at the boundary affect the motion of particles randomly, the unphysical effect less appears in the simulation result by cumulating a long time. Moreover, if the effect arising from the boundary condition is considerable in the present simulation, there would be more large number of particles in a pitch angle range with $\sim 0^{\circ}$ and $\sim 180^{\circ}$ in Fig. 10 ; this result enables us to assume that the unphysical effect at the boundary is less appeared in the present simulation.

The third is a problem of the quasi-linear diffusion coefficients in the low density plasma condition. The diffusion coefficients utilized in the estimation of the diffusion equation was calculated by using the procedure proposed by Lyons (1974) and Albert (1999). In their schemes, the high density plasma condition $\left(\omega_{p e} / \Omega_{e}\right)^{2} \gg \omega / \Omega_{e}$ was assumed. This assumption is not satisfied in the present condition where plasma parameter and wave frequency are $f_{p} / f_{c}=1.0$ and $\omega=0.3 \sim 0.6 \Omega_{e}$, respectively. Since their approximation of the dispersion relation becomes invalid for the low density plasma, diffusion coefficients can not be calculated accurately. This problem has been pointed out by Horne et al. (2003), and they developed a new calculation scheme of the diffusion coefficients by using the full dispersion relation. The recent observational evidence also suggests that the efficient energization of relativistic electrons via whistler-mode waves occurs in the low plasma density region such as the region of the outside of the plasmapause. The diffusion coefficients derived from the full dispersion scheme should be adequate to compare the simulation results in the present parameter. It is also interesting to compare the diffusion process under the condition $\left(\omega_{p e} / \Omega_{e}\right)^{2} \gg \omega / \Omega_{e}$, however, wave length of whistlermode waves and electrostatic waves become short spatial scale. This condition restrict the spatial grid size of a simulation system to be smaller than the grid size of the present simulation, time interval in the simulation is then restricted to be small enough to satisfy a numerical stability; large computational resources are required to carry out a numerical experiment with a time scale as long as the present study. Such works are important for future studies. 
The last point concerns the boundary conditions or more widely system condition adopted in the quasi-linear theory and the simulation. In the numerical experiment, we assumed that the system is open and employed open boundary conditions to treat the wave-particle interaction by injecting the whistler-mode waves from the external source into the physical region. On the other hand, in the quasi-linear theory, the diffusion of resonant particles in the velocity space is estimated under the condition that energy exchange between particles and wave field is in a quasi-equilibrium state in a closed isolated system. It is likely that the differences of the system condition may cause the difference in the estimations of the diffusion coefficients. We also think that it is necessary in the future works to examine the validity of these assumptions used in the quasi-linear theory. In such investigations, computer simulations on the basis of the present simulation technique will play an important role to understand the physics of the particle accelerations by plasma waves.

By employing the present simulation scheme, we can analyze an elementary step of the energy diffusion process and discuss the particle acceleration mechanism through the cyclotron resonance in various plasma parameters and wave modes. It will be an interesting and important subjects to investigate the resonant diffusion process in more detail by treating the phenomena under the precise plasma parameters and the more realistic system conditions.

Acknowledgments. The Electron Hybrid Code has been developed as a collaboration with Super Computing System, Information Synergy Center, Tohoku University and Research Institute for Sustainable Humanosphere (RISH) at Kyoto University. The authors would like to thank them for their consultation and advice on development of the Electron Hybrid Code. Effort of T. Ono is supported by the 21th Century COE program by the Ministry of Education, Culture, Sports, Science and Technology, Japan. The authors are pleased to acknowledge a number of helpful discussions with referees.

\section{References}

Albert, J. M., Analysis of quasi-linear diffusion coefficients, J. Geophys. Res., 104, 2429-2441, 1999.

Albert, J. M., Gyroresonant interactions of radiation belt particles with a monochromatic electromagnetic wave, J. Geophys. Res., 105, 2119121209, 2000.

Baker, D. N., J. B. Blake, L. B. Callis, J. R. Cummings, D. Hovestadt, S. Kanekal, B. Klecker, R. A. Mewaldt, and R. D. Zwickl, Relativistic electron acceleration and decay time scales in the inner and outer radiation belts: SAMPEX, Geophys. Res. Lett., 21, 409-412, 1994.
Birdsall, C. K. and A. B. Langdon, Plasma Physics via Computer Simulation, McGraw-Hill, 1985.

Blake, J. B., W. A. Kolasinski, R. W. Fillius, and E. G. Mullen, Injection of electrons and protons with energies of tens of $\mathrm{MeV}$ into $\mathrm{L}<3$ on 24 March 1991, Geophys. Res. Lett., 19, 821-824, 1992.

Brautigam, D. H. and J. M. Albert, Radial diffusion analysis of outer radiation belt electrons during the October 9, 1990, magnetic storm, $J$. Geophys. Res., 105, 291-309, 2000.

Horne, R. B., S. A. Glauert, and R. M. Thorne, Resonant diffusion of radiation belt electrons by whistler-mode chorus, Geophys. Res. Lett., 30, 1493-1496, 2003.

Kozyra, J. U., C. E. Rasmussen, R. H. Miller, and E. Villalon, Interaction of ring current and radiation belt protons with ducted plasmaspheric hiss 2. Time evolution of the distribution function, J. Geophys. Res., 100, 21911-21919, 1995.

Lyons, L. R., Pitch angle and energy diffusion coefficients from resonant interactions with ion-cyclotron and whistler waves, J. Plasma Phys., 12, 417-432, 1974.

Lyons, L. R. and R. M. Thorne, Equilibrium structure of radiation belt electrons, J. Geophys. Res., 78, 13, 2142-2149, 1973.

Meredith, N. P., R. B. Horne, D. Summers, R. M. Thorne, R. H. A. Iles, D. Heyndreickx, and R. R. Anderson, Evidence for acceleration of outer zone electrons to relativistic energies by whistler mode chorus, Ann. Geophys., 20, 967-979, 2002.

Miyoshi, Y., A. Morioka, T. Obara, H. Misawa, T. Nagai, and Y. Kasahara, Rebuilding process of the outer radiation belt during the 3 November 1993 magnetic storm: NOAA and EXOS-D observations, J. Geophys. Res., 108, 1004-1018, 2003.

Obara, T., T. Nagatsuma, M. Den, Y. Miyoshi, and A. Morioka, Mainphase creation of "seed" electrons in the outer radiation belt, Earth Planets Space, 52, 41-47, 2000.

Omura, Y. and H. Matsumoto, Computer simulations of basic process of coherent whistler wave-particle interactions in the magnetosphere, $J$. Geophys. Res., 87, 4435-4444, 1982.

Omura, Y. and H. Matsumoto, KEMPO1: Technical guide to onedimensional electromagnetic particle code, in Computer Space Plasma Physics: Simulation Techniques and Softwares, edited by H. Matsumoto and Y. Omura, Terra Scientific Pub., Tokyo, 1985.

Rathmann, C. E., J. L. Vomvoridis, and J. Denavit, Long-time-scale simulation of resonant particle effects in Langmuir and whistler waves, $J$. Computational Phys., 26, 408-442, 1978.

Roth, I., M. Temerin, and M. K. Hudson, Resonant enhancement of relativistic electron fluxes during geomagnetically active periods, Ann. Geophys., 17, 631-638, 1999.

Selesnick, R. S. and J. B. Blake, On the source location of radiation belt relativistic electrons, J. Geophys. Res., 105, 2607-2624, 2000.

Summers, D. and C. Y. Ma, A model for generating relativistic electrons in the Earth's inner magnetosphere based on gyroresonant wave-particle interactions, J. Geophys. Res., 105, 2625-2639, 2000.

Taguchi, T., J. Thomas, M. Antonsen, C. S. Liu, and K. Mima, Structure formation and tearing of an $\mathrm{MeV}$ cylindrical electron beam in a laserproduced plasma, Phys. Rev. Lett., 86, 5055-5058, 2001.

Vampola, A. L. and A. Korth, Electron drift echoes in the inner magnetosphere, Geophys. Res. Lett., 19, 625-628, 1992.

Y. Katoh (e-mail: yuto@rish.kyoto-u.ac.jp), T. Ono, and M. Iizima 